\title{
Globalización y competitividad. Desafios para las ciudades centroamericanas y caribeñas 1
}

\section{Introducción: sobre la globalización y la competitividad urbana}

Sin entrar a realizar un análisis detallado del carácter de la globalización actualmente en curso, sus múltiples dimensiones y sus discutibles consecuencias, no puede eludirse la imperiosa necesidad, cualquiera que sea la opción de desarrollo que se impulse, de incrementar los niveles de productividad de la economía de las ciudades. No hacerlo implica asumir los costos negativos de la ineludible globalización, que recaen obligatoriamente sobre la población excluida socialmente y de menores ingresos. Para hacerlo, es necesario ir más allá de la estricta dimensión económica de la problemática de la competitividad. Explorar este camino es el objetivo de este trabajo, que prolonga las reflexiones de otro elaborado hace algunos años (Lungo, 1993).

Efectivamente, y aunque se debe partir del incremento de la productividad de la economía urbana (Cohen, 1991), como una base indispensable para elevar la competitividad de las ciudades, esta incluye además, en nuestra opinión, otras dos dimensiones indisolublemente ligadas a la primera: el mejoramiento de la calidad de vida de la población y la construcción de nuevas relaciones de gobernabilidad urbanas de carácter democrático.

Alrededor de estas tres dimensiones giran otras cuestiones abordadas frecuentemente en la literatura sobre el desarrollo urbano: la pobreza, la exclusión social, la segregación socio-espacial, el atraso tecnológico, la degradación ambiental urbana, la participación social, la descentralización, el desarrollo económico local, etc. Pero existen relaciones con otras cuestiones que no aparecen en un primer plano, como la actual estructura social que se ha ido configurando en las ciudades centroamericanas y caribeñas, o las nuevas identidades urbanas que están en la base de la estructuración de los actores sociales urbanos que cotidianamente construyen nuestras ciudades. 
La competitividad comprende, entonces, múltiples y complejas relaciones que se tejen por estos actores alrededor de las tres dimensiones básicas descritas anteriormente. El esquema que se presenta más adelante intenta captar esta idea. Precisemos antes una diferencia que consideramos esencial entre los grupos portadores de intereses (stakeholders en la literatura inglesa) y los actores sociales. Planteamos que los primeros tienen un carácter fundamentalmente corporativo, lo que hace que sus intereses no se inscriban necesariamente en un proyecto de desarrollo global de la ciudad, mientras que los segundos, por el contenido social y político de sus acciones, tienden a constituir parte de una opción de desarrollo que desborda sus intereses propios y, por eso mismo, trascienden lo inmediato. Lo anterior es clave a la hora de la construcción de una estrategia que pretenda elevar la competitividad de una ciudad de forma sostenible.

Abordar aisladamente el conjunto de cuestiones que aparecen en el esquema anterior, tanto en el aspecto analítico como en lo referente a la formulación de políticas, constituye una limitación para lograr la elevación de los niveles de competitividad de las ciudades. Tal es el caso de las acciones aisladas que buscan reducir la pobreza urbana, lo que ha sido ampliamente reconocido incluso por los organismos de cooperación internacional (Ayres, 1997; Bustelo y Minujín, 1997), el mundo académico y las organizaciones sociales y políticas, avanzándose sustancialmente hacia la utilización de enfoques más integrales como la vulnerabilidad social (Moser, 1996) y la exclusión social, que incorpora las prácticas y los derechos sociales constitutivos de la integración social y, por lo tanto, identidad (FLACSO/PRODERE, 1995).

Lo mismo ocurre con la incorporación de la problemática ambiental urbana a partir de una visión más amplia (Bartone, 1994; Serageldin, et al., 1994), que ha incorporado incluso de manera novedosa los riesgos a desastres urbanos, un tema no relacionado antes con la competitividad (Lavell, 1993). En este aspecto se ha avanzado sustancialmente durante los últimos años.

Otras de las cuestiones que han experimentado cambios en su abordaje son la participación social y la descentralización, en la medida en que se han analizado a partir de una problemática que las engloba y que ha cobrado fuerza también en los últimos años: la gobernabilidad urbana, y que ha sido definida como la relación entre el Estado y la sociedad civil y no meramente como una problemática del gobierno en sí mismo, lo que modifica sustancialmente cuestiones como la participación social en la gestión del desarrollo de las ciudades (McCarney et al., 1995; Lungo, 1998).

No obstante la tendencia descrita a integrar problemáticas que se han enfocado anteriormente de manera separada, hay pendientes aún dos cuestiones cruciales para la competitividad urbana, que son precisamente los que tienen un alto contenido subjetivo y por eso permanecen en un segundo plano: las nuevas estructu- 
ras sociales e identidades urbanas que se han ido configurando en las ciudades centroamericanas y caribeñas en los años de la nueva globalización.

En un trabajo reciente planteamos que la construcción de una gobernabilidad urbana democrática, particularmente a nivel metropolitano (que es el caso de la mayoría de las principales ciudades de Centroamérica y el Caribe), requiere no sólo de la existencia de una adecuada forma de gobierno de la ciudad y la más amplia participación social, sino también de la existencia de identidades urbanas $y$, en este caso, de identidades metropolitanas fuertes, y debe promoverse su constitución en los casos en que éstas no existan o sean débiles (Lungo, 1998b). No obstante las limitadas señaladas al abordaje sectorial y aislado de todas estas cuestiones, la globalización es un proceso muy complejo y demasiado general que dificulta su utilización en términos analíticos. Por la razón anterior es que proponemos la competitividad urbana (entendida en el sentido amplio antes descrito) como una categoría intermedia útil para los objetivos de este análisis. Este enfoque metodológico ha sido aplicado en otras investigaciones urbanas (Portes, 1997).

\section{La situación en los años noventa}

Como hemos sostenido en un trabajo anterior sobre la relación entre globalización y desarrollo urbano en las ciudades centroamericanas y caribeñas (Lungo, 1993), a inicios de los años noventa señalábamos varias tendencias importantes que persisten al finalizar el siglo:

(a) Respecto al crecimiento demográfico, la primacía urbana no seguía una tendencia uniforme, aumentando en unos países pero disminuyendo en otros, notándose también evoluciones disímiles respecto a las redes urbanas nacionales. Esta cuestión se vincula a la competitividad en la medida en que un punto muy discutible es el que gira en torno a las deseconomías que genera una excesiva concentración poblacional. En el caso de los países que nos ocupan, por su relativamente bajo nivel de urbanización y el tamaño de las principales ciudades, pareciera que no se enfrenta una situación crítica que constituya un obstáculo para el aumento de la competitividad de las mismas.

(b) Por el contrario, el carácter inequitativo de la distribución de los recursos y la riqueza generada por las economías urbanas, además del alto grado de informalización, desempleo y el atraso tecnológico de éstas, sí se revelaban como factores negativos para hacer más competitivas las ciudades de la región.

(c) La segregación socio-espacial, con sus diversas expresiones, también aparecía como un factor negativo al contribuir a la exclusión social. 
Cuando revisamos este trabajo al final de esta década, es evidente que quedaron fuera del análisis hecho varios aspectos esenciales sobre la temática de la competitividad. El primero de ellos se refiere a las características del marco construido (la infraestructura, los equipamientos y la vivienda). El segundo es la cuestión del medio ambiente en su doble rostro: por un lado, la capacidad de soporte de los bienes y servicios ambientales con que cuenta una ciudad; por otro, los niveles de contaminación que se producen y la capacidad de absorción de la misma. El tercero, la gobernabilidad urbana.

Observando lo ocurrido en las ciudades centroamericanas y caribeñas durante la segunda mitad de esta década podemos plantear la hipótesis de que la situación de estos tres últimos áspectos, sumado a los factores negativos de los primeros, no permite ser optimista respecto a la competitividad de estas ciudades. Es cierto que en varias de ellas los esfuerzos por modernizar la infraestructura son palpables y que la conciencia sobre la degradación del medio ambiente urbano y los costos sociales y económicos de la misma es creciente, pero no se encuentran innovaciones profundas y sostenibles en términos de la construcción de nuevas relaciones de gobernabilidad urbana democrática, lo que se suma a la persistencia de altos niveles de pobreza y exclusión social.

Como decíamos anteriormente, a pesar de la concentración poblacional en las principales ciudades, en varios países estamos en presencia de un crecimiento de las ciudades secundarias, producida en algunos casos por el desarrollo del sector turístico, como en la República Dominicana, o de la maquila, como en Honduras, lo que se refuerza por los procesos de descentralización e impulso del desarrollo local, a pesar de las dificultades que éstos enfrentan en muchos países (Campbell, 1997), por lo que reiteramos que la concentración de la población urbana no es en sí misma un factor negativo ni se está acercando a umbrales críticos.

La pobreza urbana, a pesar de que en algunos casos ha disminuido, sigue siendo, quizás, el factor negativo de mayor peso. Aun en el caso de la ciudad de San José, en Costa Rica, país que presenta la menor desigualdad económica y social en Centroamérica, es perceptible el crecimiento de la informalidad urbana y el desempleo (Tardanico y Lungo, 1997), aunque a pesar de la crisis fiscal de mediados de los años noventa se han logrado mantener condiciones sociales y económicas relativamente buenas que han permitido, apoyándose en una fuerza de trabajo capacitada y adiestrada en tecnologías modernas, competir exitosamente por la localización de empresas extranjeras con tecnología de punta en la ciudad.

No contamos con estudios detallados sobre la exclusión social en las ciudades, tal como se ha hecho a nivel departamental para tres países centroamericanos (FLACSO/PRODERE, 1995), pero uno de los factores componentes de la misma, las distintas formas de la segregación socio-espacial, son visibles en todas las ciudades. Particularmente nos referimos aquí no sólo a sus expresiones más tradicionales, los grandes asentamientos marginales, sino al creciente surgi- 
miento de conjuntos cerrados destinados especialmente para sectores sociales de altos ingresos, fenómeno común en toda América Latina.

La infraestructura y el equipamiento muestran, además de un alto grado de obsolescencia en muchas ciudades, una distribución y un acceso altamente desigual en términos sociales, que contribuye a reducir la productividad de la economía, deteriorar las condiciones de vida de la población y acentuar la degradación del medio ambiente. Los costos provocados por los accidentes de tránsito debido a las malas condiciones de la red vial, en Managua han ido en aumento en la medida en que estos se incrementaron en un 15 por ciento entre 1992 y 1994 (Avilés, 1998).

Respecto a la degradación ambiental urbana, una investigación sobre San Salvador mostró la gravedad de la situación actual e, indirectamente, permitió observar su negativa influencia en los niveles de competitividad de esta ciudad centroamericana (Lungo y Oporto, 1998).

En torno a la cuestión de la gobernabilidad urbana, el caso de la República Dominicana muestra la importancia de los recientes procesos de descentralización en la construcción de nuevas relaciones entre los gobiernos locales y las organizaciones de la sociedad civil (Yunén, 1997).

Las ciudades centroamericanas (y esta característica podría extenderse a las ciudades caribeñas) están espacialmente desarticuladas y profundamente divididas desde el punto de vista social, son inseguras y cumplen sólo mínimamente las funciones centrales para asegurar el funcionamiento eficiente de las distintas actividades de la ciudad, particularmente las economías de escala (Polese, 1998). En términos generales, se pueden visualizar las siguientes tendencias generales en las economías urbanas de las principales ciudades de la región al finalizar el siglo: (a) terciarización creciente con predominio del sector financiero; (b) crecimiento del sector informal mientras se complejizan sus relaciones con el sector formal; (c) incremento de la desigualdad en la distribución de la riqueza, aunque los niveles de pobreza urbana disminuyan; (d) surgimiento de nuevos patrones de localización de las actividades; (e) cambios en las relaciones con otras ciudades de las redes urbanas nacionales, y $(f)$ la constitución de nuevos actores económicos urbanos.

A las tendencias anteriores hay que sumar que el proceso de globalización, sumado a la pequeña dimensión de las economías de estos países, induce al desbordamiento de las fronteras nacionales en el caso de muchas de las funciones urbanas. Un ejemplo al respecto es el caso de San Salvador, ciudad que constituiría, para muchos grupos empresariales, una plataforma económica para desplegar sus actividades a nivel centroamericano. Ya en los años sesenta, un análisis comparativo mostraba que el Área Metropolitana de San Salvador tenía la mayor dotación de infraestructura y servicios entre las capitales centroamerica- 
nas; su producción industrial doblaba a la de la ciudad de Guatemala, la más poblada en ese momento en la región; y el potencial de mercado y empleo (en base a la unidad tiempo/distancia) mostraba que, en San Salvador, el 27 por ciento de la población se encontraba en su radio de influencia, contra una proporción del 25 por ciento en Guatemala y 12 por ciento en Tegucigalpa, la capital de Honduras (CONAPLAN, 1969).

El panorama descrito brevemente no es alentador, mientras es innegable que el proceso de globalización económica, social y cultural continua avanzando con fuerza, potenciado, en la mayoría de los países de Centroamérica y el Caribe, por un fenómeno cuyas implicaciones sobre el desarrollo de las ciudades y sus niveles de competitividad aún no ha sido analizado: la migración internacional (Lungo, 1994). No obstante hay que tener presente que los procesos de urbanización, además de su larga temporalidad, son de una extrema complejidad y están cruzados por condicionalidades extraeconómicas, por lo que no se debe plantear que la globalización de la economías se traduce directamente en la globalización de los procesos de urbanización.

\section{Los desafios y las estrategias para incrementar la competitividad de las ciudades}

Ante esta situación, que muestra tendencias negativas similares a muchas de las que experimentan las ciudades de los países desarrollados: el crecimiento del desempleo, el deterioro de la infraestructura, la degradación del medio ambiente, la desintegración de las relaciones sociales y la debilidad de las instituciones encargadas de la gestión urbana (Cohen, 1996) es imperativo superarlas, ya que constituyen el mayor desafío para aumentar los niveles de competitividad de nuestras ciudades en este momento de globalización.

La primera de estas tendencias negativas gira alrededor del doble fenómeno del desempleo y el crecimiento del sector informal, donde pocos ejemplos de soluciones sostenibles se encuentran en las ciudades de la región. El modelo actual prioriza empleos de baja calificación técnica en la mayoría de sectores de la economía, salvo en los servicios a las empresas y el sector financiero, pero que constituyen una proporción minoritaria y, por lo mismo, acentúan la segmentación de los mercados laborales urbanos. La estrategia, aunque clara, no parece fácil de impulsar: la creación de empleos permanentes de cada vez mayor calificación en sectores que resistan a la volatilidad de los movimientos financieros internacionales.

El deterioro de la infraestructura tiene también un doble rostro: se trata de la infraestructura que sirve de soporte a los procesos productivos, pero también de la infraestructura necesaria para la reproducción social. En algunos casos, el énfasis ha sido puesto en mejorar las condiciones de vida de los sectores sociales más pobres de las ciudades, dejando en un segundo plano las necesidades de 
la producción. Más recientemente, el énfasis parece estarse invirtiendo, corriéndose el riesgo de caer en la posición extrema opuesta. Adicionalmente, la urgencia de la modernización de la infraestructura urbana no debe hacer olvidar sus relaciones con el medio ambiente y la debilidad en la capacidad de gestión en este sector.

La degradación del medio ambiente urbano y sus costos económicos y sociales ya han sido ampliamente reconocidos. Permanece, no obstante, la difícil tarea de conciliar el uso de los recursos y servicios ambientales que ofrece una ciudad con las necesidades de su crecimiento. Aquí aparece con claridad la urgencia de retomar el tema de la gestión del suelo urbano y vincularlo a la sostenibilidad y a la competitividad.

La segmentación que caracteriza a las actuales estructuras sociales urbanas, y sobre todo la creciente exclusión social y sus nuevas modalidades, también comienza a llamar la atención de los investigadores y los formuladores de políticas. Cuestiones como la importancia del capital social adquieren cada día mayor importancia para enfrentar integralmente la pobreza urbana y la exclusión social.

Enfrentar las debilidades de las instituciones responsables de la gestión del desarrollo urbano exige ir más allá de la preocupación por el establecimiento de un buen gobierno y de la "accountibility". Es indispensable, como sosteníamos al inicio, construir nuevas relaciones de gobernabilidad urbana de carácter democrático.

Finalmente, queremos apoyamos en las propuestas presentadas en un artículo sobre las posibles estrategias urbanas para hacer frente a la globalización (Savitch, 1996):

(a) Las ciudades deben reconocer que el desarrollo inducido por los gobiernos tienen serias limitaciones, por lo que es indispensable incorporar en el mismo a la sociedad civil.

(b) En términos de aumentar su competitividad, las ciudades deben aprovechar sus ventajas naturales, lo que demanda hacer análisis integrales desde una nueva óptica metodológica, como se ha ensayado para el caso de San Salvador (Cuervo, 1998).

(c) Las ciudades deben recrear sus relaciones con otras ciudades, tanto nacionales como internacionales, y sus relaciones internas.

(d) La gestión de las ciudades debe promover la creación de las expresiones más diversas de capital social (Portes,1998), especialmente sus expresiones urbanas.

(e) Es indispensable, además, incorporar de manera integral y decisivamente la dimensión de las expresiones culturales urbanas, cuestión que comienza a ser el foco de atención incluso de instituciones antes ajenas a esta 
problemática, como el Banco Mundial, el cual plantea que la autoestima y el orgullo que provienen de la identidad cultural son componentes esenciales para el "empowerment" de las distintas comunidades (The World Bank, 1998).

\section{Nota}

1. Ponencia presentada en el Seminario Internacional "Ciudades abiertas, ciudades competitivas", 6 Encuentro Internacional Hábitat Colombia, octubre, 1998.

\section{Referencias bibliográficas}

Avilés, Isabel. (1998). "El financiamiento de la infraestructura vial en Managua". En Economía y desarrollo urbano en Centroamérica, Mario Lungo y Mario Polese (compiladores), FLACSO IGIM, San José.

Bartone, Carl, et al. (1994). Toward Enviroimiental Sirategies for Cities, Urban Manage iietit Program, The World Bank, Washington.

Campbell, Tim. (1997). Innovations and Risk Taking. The Engine of Reform in Local Government in Latin America and the Caribbean, The World Bank, Washington.

Cohen, Michael. (1991). The Urban Agenda for the 90's, The World Bank, Washington.

Cohen, Michael. (1996). "The Hypothesis of Urban Convergence: Are Cities in the North and South Becoming More Al Ike in the Age of Globalization?". En Preparing for Urban Future, M.

Cohen, et al. (eds.). The Woodrow Wilson Center, Washington.

CONAPLAN. (1969). Metroplan 80, San Salvador.

Cuervo, Luis Mauricio. (1998). Dinámica económica de la región metropolitana de San Salvador, PRISMA, San Salvador.

FLACSO 1 PRODERE. (1995). Análisis de la exclusión social a nivel departamental. Los casos de Costa Rica, El Salvador y Guatemala, FLACSO, San José.

Lavell, Alían. (1993). "Prevención y mitigación de desastres en Centroamérica y Panamá". En Desastres y Sociedad, 1, La RED, Lima.

Lungo, Mario. (1993). "Las ciudades y la globalización. Una mirada desde Centroamérica y el Caribe". Revista Interamericana de Planificación, 104, San Antonio, Texas.

Lungo, Mario. (1994). "Impacts of International Migration on Cities". En Urban Age, The World Bank, Washington.

Lungo. Mario. (1998a). "Los planes de desarrollo urbano. Construyendo nuevas relaciones de gobemabilidad urbana". En Gobernabilidad urbana en Centroamérica, FLACSO 1 GURI, San José.

Lungo, Mario. (1998b). "Metropolitan Planning and Civil Society Participation: Developing Urban Governance Relations in El Salvador": Ponencia presentada coloquio MOST IC MC, Ciudad del Cabo, Sudáfrica.

Lungo, Mario. (1998c). "Planificación urbana y economía en las ciudades centroamericanas". En Economia y desarrollo urbano en Centroamérica, M. Lungo y M. Polese (compiladores), FLACSO/GIM, San José.

Lungo, Mario y Oporto, Francisco. (1998). "Construyendo una estrategia ambiental para la región metropolitana de San Salvador". En Realidad, 65, San Salvador. 
McCarney, Patricia, et al. (1995). "Towards an Understanding of Governance. The Emergence of an Idea and its Implications for Urban Research in Developing Countries". En Perspectives on the City, Richard Stren y Judith Belí (eds.), University. of Toronto.

Bustelo, Edurado y Minujín, Alberto. (1997). "La política social esquiva". En Pobreza, exclusión y política social, R. Menjívar, et al. (editores), FLACSOIU de UtrechtI UNESCO-MOST, San José.

Moser, Caroline. (1996). Con fronting Crisis. A comparative Study of Household Responses to Poverty and Vulnerabllity in Four Poor Urban Communities, The World Bank, Washington.

Polese, Mario. (1998). "Ciudades y empleos en Centroamérica: elementos para el diseño de estrategias de desarrollo económico con base urbana". En Economía y desarrollo urbano en Centroamérica, M. Lungo y M. Polese (compiladores), FLACSO 1 GIM, San José.

Portes, Alejandro, et al. (1997). The Urban Caribbean, The Jonhs Hopkins University Press, Baltimore.

Portes, Alejandro. (1998). "Social Capital: its Origins and Applications in Modern Sociology". En Annual Review of Sociology.

Savitch, H. V. (1996). "Cities in a Global Era: A New Paradigm br the Next Millenium?". En Preparing for Urban Future, M. Cohen, et al. (editors). The Woodrow Wilson Center, Washington.

Serageldin, Ismail, et al. (1994). The Human Face of of the Urban Environment, The World Bank, Washington.

Tardanico, Richard y Lungo, Mario. (1997). "Continuities and discontinuities in Costa Rica Urban Employment". En Global Restructuring, Employment and Social lnequality in Latin America, R. Tardanico y R. Menjivar (eds.), North South Center Press, Miami.

The World Bank. (1998). Culture and Development at the Millennium. The Challenge and the Response, Washington.

Yunén, Rafael. (1997). Gobernabilidad urbana en el Caribe, PUCMM, Santiago de los Caballeros. 